\title{
On-line control of moving masks and windows on a complex background using the ATVista videographics adapter
}

\author{
PAUL M. J. VAN DIEPEN, PETER DE GRAEF, and JOHAN VAN RENSBERGEN \\ University of Leuven, Leuven, Belgium
}

\begin{abstract}
In reading research, the moving mask and moving window paradigms have proved to be invaluable in determining the chronometric and spatial characteristics of processing written text. The success of these methods has lead to a demand for their application in research on real-world scene perception. However, we will argue that the technical implementation of eye-contingent mask (window) movement across a stable text cannot be applied to scene research. A new technique is proposed that allows graphical masks or windows of arbitrary form, size, and content to be moved quickly over a complex graphical stimulus. This moving overlay technique makes use of the ATVista graphics adapter, a board with the ability to mix an internally stored and an externally generated image into one composite image. A high-performance moving mask or window is created by programming the internal image to be movable and partly transparent. The technique is implemented on a standard personal computer interfaced with an eyetracker, thus bringing mask (window) movement under online eye-movement control. We discuss general principles of the technique and illustrate them with performance data from a concrete experimental setup.
\end{abstract}

A powerful paradigm used in reading research involves the presentation of a visual mask or window, moving across the text in synchrony with the eyes. Thanks to this paradigm, the chronometry and spatial extent of information extraction in reading are better understood (for a review, see Rayner \& Pollatsek, 1989, chapter 4). Recent research on real-world scene perception has demonstrated the potential of eye-movement registration in studying the recognition and comprehension of complex pictorial information (Boyce \& Pollatsek, 1992; De Graef, 1992; De Graef, De Troy, \& d'Ydewalle, 1992). Until now, however, technical difficulties have prevented the full exploitation of eye-movement methodology in the study of scene perception. Specifically, the application of the moving window and/or moving mask techniques has proved to be quite cumbersome when realistic, fullcolor, high-resolution images are used as experimental

This research was supported by a Concerted Research Action (G.O.A.) of the University of Leuven and by agreement RFO/Al/04 of the Incentive Program for Fundamental Research in Artificial Intelligence. The work was conducted while P.M.J.v.D. was a student at the Nijmegen Institute for Cognition and Information, University of Nijmegen, the Netherlands, and held an Erasmus scholarship at the Laboratory of Experimental Psychology, Leuven, Belgium. The authors are indebted to Andreas De Troy and Noël Bovens for their assistance in implementing the eyetracking infrastructure. Further thanks to Charles de Weert, Géry d'Ydewalle, A. Finley, Karl Verfaillie, and two anonymous reviewers for their comments on an earlier draft. Correspondence may be addressed to P. M. J. van Diepen or P. De Graef at the Laboratory of Experimental Psychology, University of Leuven, Tiensestraat 102, B-3000 Leuven, Belgium (e-mail: paul. vandiepen@psy.kuleuven.ac.be or peter.degraef@psy.kuleuven.ac.be). stimuli. In the present article, we will describe the nature of the encountered difficulties and will present a new display change technique that is able to clear away these obstacles.

At the most rudimentary level of description, any eyemovement pattern across a static display consists of a series of fixations and saccades. A fixation is a period during which the eyes are in rest and information is being extracted from the stimulus. A saccade is a ballistic eye movement executed to jump from one fixation position to another. During a saccade, visual resolution diminishes and sensitivity to stimulus changes decreases considerably (Ishida \& Ikeda, 1989; Matin, 1974). This basic characterization of eye movements sets the stage for two types of eye-contingent display changes that have been used extensively in reading research. In the moving window technique, all text within a window of $n$ characters around the point of fixation is left unaltered while textual information outside this window is changed in various ways. Whenever the eyes move to a new fixation position, the window is shifted to that new position during the saccade (McConkie \& Rayner, 1975). Consequently, if reading is somehow disturbed under these windowing conditions, one can infer that the changed information outside the window is instrumental to normal reading performance. This technique has been particularly useful in determining the spatial extent of text processing. The moving mask technique is especially suited for examining the chronometry of foveal information extraction. By masking an area at the fixation position, and by manipulating the mask onset delay from the start of the fixation, one can gain insight in the speed with 
which foveal information is acquired (Rayner, Inhoff, Morrison, Slowiaczek, \& Bertera, 1981).

Obviously, a first requisite for both techniques is the accurate, on-line discrimination of fixations and saccades, as well as the precise localization of fixation position. An adequate solution to these problems depends critically on the sampling speed of the eyetracker and the quality of the algorithm for identifying periods of eye movement and eye stability. An extensive description of the eyetracking infrastructure underlying our proposal can be found in Van Rensbergen and De Troy (1993). In the present text we will concentrate on a second requirement, the fast and precisely timed displaying of the appropriate mask or window at the appropriate position.

In reading research, fulfilling this second requirement is easier than it is in the study of scene perception. First, in normal left-to-right reading, the eye-movement pattern is sufficiently regular to anticipate the next fixation position. Hence, the mask (window) can already be shifted to the appropriate position during the eye's saccade toward that location. While saccades in reading typically only last on the order of $20-35 \mathrm{msec}$ (Rayner \& Pollatsek, 1989), this saccadic interval is generally sufficiently long to complete the desired text changes. In free scene exploration, however, the unpredictability of eye movements is such that the appropriate position to display the mask (window) can only be determined when the eye is already there. Second, text is a relatively simple sort of stimulus to present: a standardized set of easy-to-position, monochrome characters. Consequently, text is amenable to presentation on a vector display allowing for very fast stimulus changes. In fact, the speed of vector displays is such that a relatively simple line drawing of a scene can be changed in under $4 \mathrm{msec}$ (Boyce \& Pollatsek, 1992). Unfortunately, vector displays are quite expensive, are traditionally driven by a mini- rather than a microcomputer, and are very limited in terms of the stimuli that can be displayed: Full-color stimuli are out of the question, and no easy transfer of the output of graphics software onto the vector display is available. These difficulties can be solved by opting for a raster display, which, however, imposes a strong limit on the speed of stimulus change, or the refresh rate of the display (between 50 and $100 \mathrm{~Hz}$ for affordable models). Third, even under the restrictions imposed by the use of a raster display, fast text changes remain conceivable because, usually, only small portions of text are changed. Moreover, complete letters are stored in single bytes. In contrast, full-color, high-resolution images require 1 or more bytes per pixel, which implies that changing these images involves much larger amounts of transfer to and from the video memory on the graphics board.

Several methods have already been proposed for increasing the speed of complex display changes. However, these methods fall short of the requirements for the use of the moving window or mask techniques in scene perception. Probably the best known method in visual perception research is the virtual screen technique (see, e.g., Finley, 1991). Stimuli are prepared and assigned to several, distinct pages of video memory. Following selection of one page as the active page, the stimulus in that page is written to the display in one screen refresh. Note that the number of stimuli that can be stored for such rapid presentation is strictly limited by the number of pages that can be defined in video memory.

Wittebrood, Wansink, and de Weert (1981) and, more recently, Dixon (1991) describe an alternative displaychange technique that makes use of the color lookup tables (LUTs) that are currently implemented on many graphics boards. An LUT maps pixel values contained in the video memory onto hardware colors. If one simply changes the content of one entry in the table, all pixels pointing to that entry will appear on the screen in the newly assigned color. For instance, suppose that on frame $n$ one wishes to present a green background with a red rectangle on it, and that on frame $n+1$ the red rectangle needs to be replaced by a nonoverlapping blue circle. This can be achieved by assigning different pixel values to the rectangle (value 1), the circle (value 2) and the background (value 3 ). LUT entry 1 can then be mapped onto red on frame $n$ and onto green on frame $n+1$, while entry 2 first maps onto green and then onto blue, and entry 3 maps onto green during both frames. With this technique, the potential complexity of stimuli and stimulus changes is limited by the number of available LUT entries: overlap between distinct stimulus components, use of multichromatic components, partial change of stimulus components-all require additional LUT entries.

Although both techniques allow for fast display changes, clearly their major drawback is that for every possible fixation position on the screen a separate stimulusplus-mask or stimulus-plus-window configuration has to be prepared in advance. Since in most scene perception research every portion of the screen may be selected for fixation, and this in a highly unpredictable pattern, too many prepared stimuli would be necessary to cover all possible situations. Hence, virtual screen or color LUT techniques will only suffice when the task strictly constrains the viewer's eye-movement pattern.

An alternative to the clever programming of standard graphics boards is to turn to customized hardware solutions. Saida and Ikeda (1979) developed a moving window technique where a camera image of a bright square gated the camera image of a stimulus. The square was generated on an oscilloscope by a function generator, and its position was directly contingent on the position of the viewer's eye. Although this technique can be quite fast and it allows for the presentation of complex stimuli within the window, one can only blank the display outside the window. More subtle changes of visual information are impossible, barring investigation of the kinds of information that can be extracted at different distances from fixation. Similarly, while this technique could probably be modified to produce a moving mask, 
this would inevitably consist of a rectangular, blanked area and thus prevent the use of more effective pattern masks or conceptual masks (Loftus \& Ginn, 1984).

We now turn to a description of our own method for realizing fast, precisely timed and positioned eyemovement-contingent display changes in high-resolution, full-color images.

\section{THE MOVING OVERLAY METHOD}

Early reading research (Poulton, 1962) used a simple but somewhat primitive moving window technique: A mask with a hole in it was moved over a "hard-copy" text. In essence, the technique presented here is the electronic equivalent of that first straightforward method. The implementation of the technique is centered on the ATVista videographics adapter (henceforth called the Vista). The Vista, a product of Truevision, Inc., is a graphics board for use in a standard IBM-AT-compatible microcomputer (see Truevision, 1988). A version for Apple Macintosh is also available under the name $\mathrm{Nu}-$ Vista. The board contains video and instruction memory, and a Texas Instruments TMS 34010 graphics system processor (see Texas Instruments, 1986). Several types of Vista boards are available at prices around $\$ 4,500$ (for Europe), depending on the amount of memory and on the processing speed. All applications discussed in this paper were implemented on a Vista with $4 \mathrm{Mb}$ of video memory, $4 \mathrm{Mb}$ of processor memory, and a $40-\mathrm{MHz}$ processor clock. Programming of the board was done in Turbo C 2.0, using the Standard Truevision Adaptable Graphics Environment (STAGE, Truevision Inc., 1989). All Vista boards have four video data channels with corresponding inputs and outputs: red (R), green $(G)$, blue (B), and a general-purpose channel (Alpha). The Vista video inputs can take an externally generated analog video image (e.g., the image from a camera or a VCR) and convert it to digital data. Thus, two image sources are available for video output: an external image generated off the board, and an internal image stored in the on-board video memory (e.g., an image created with a graphics software package). The Vista supports a wide range of user-programmable video modes, including the frequently used NTSC and PAL (both interlaced and noninterlaced).

From this brief description, it should be clear that the Vista has a quantitative advantage over other graphics adapters. The large amounts of video memory in combination with a dedicated on-board graphics processor make it possible to use the board as a powerful multichannel tachistoscope. For instance, in NTSC 8-bit video mode, as many as 10 virtual screens with a $756 \times 486$ resolution can be stored simultaneously in video memory, while switches between screens are fast and can be precisely timed (see below). Moreover, the architecture of the board also includes color LUTs with as many as $\mathbf{2 5 6}$ different entries, which enables sophisticated display-change techniques. However, apart from these quantitative advantages, the special input-output architecture of the Vista constitutes a qualitative advantage that underlies the new display-change technique described in this paper.

As mentioned above, two video sources are simultaneously available for Vista output (Figure 1). Which source is output depends on the selected display mode. Three main categories of display modes can be distinguished: live modes, stored modes, and overlay modes. In live mode, only the external image is output. In stored mode, the internal image is output. In overlay mode, either image can be output, depending on the values stored for every individual pixel in the internal image. This makes it possible to use an external image as background and to superimpose an internal image on top of it that can be rendered transparent at any desired position in the image. This can be explained best with reference to the 16-bit overlay independent mode. In this mode, R, G, and B channels can be modified independently. The data for an individual pixel in this mode consist of 5 bits per channel, allowing for the selection from a palette of $32 \times 32 \times 32$ colors, which is quite sufficient for detailed colored pictures. The 16th bit offers control over the transparency of the pixel defined in the 15 other bits: When set to 0 , the pixel is opaque; when

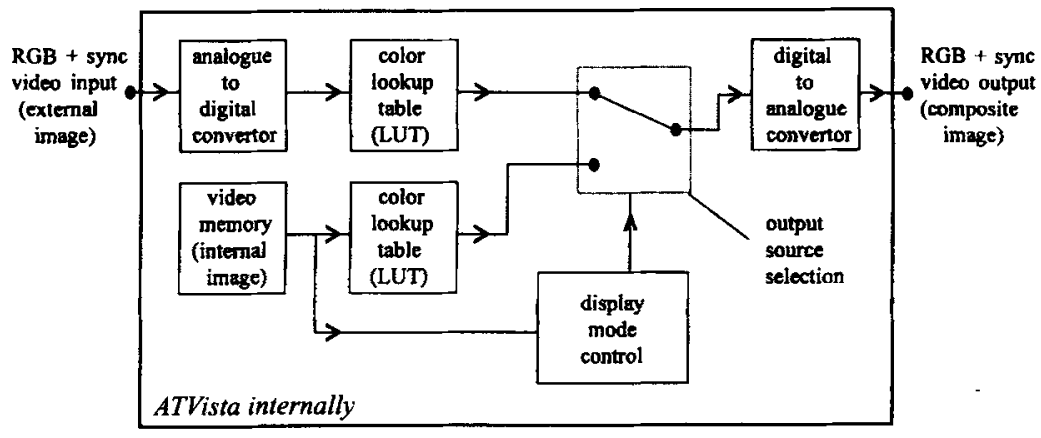

Figure 1. Block diagram of the Vista video architecture, capable of outputting images from either an external or an internal source. 
set to 1 , it becomes completely transparent. Importantly, pixel bits can be write-protected, making it possible to alter the internal image without changing the transparency bit, and vice versa. When the Vista is operating in stored mode, the 16th bit is taken to carry alpha channel data rather than transparency information. Since our application does not monitor the alpha channel, the very fast process of switching between stored and overlay modes is tantamount to switching between an opaque internal image and an internal image that is transparent at all the predesignated pixels where it offers a view of the external image. A switch to live mode completely disables the internal image and leaves only the external image. ${ }^{1}$

Clearly, if the transparency of the internal image could be made contingent on fixation position, we would have implemented a moving mask or window across a stable high-resolution, full-color image. Our solution to this problem is based on the distinction between displayable resolution and addressable resolution. Every video mode is characterized by a particular spatial resolution of the pixel matrix that it outputs to the screen: the displayable resolution. The Vista also offers a userprogrammable spatial resolution of the on-board video memory: the addressable resolution. The addressable resolution has to be at least equal to the displayable resolution, but it can be bigger. In this case, only a part of the addressable video memory can be displayed on the screen. This part is determined by the user, who can point an imaginary camera at any part of the addressable video memory. The Vista allows changes in "camera position" (or "panning") with a 1-pixel resolution. As is illustrated in Figure 2, this implies that any pixel of the addressable video memory can be designated as the upper left corner of the matrix displayed on the screen, provided that the lower right corner remains within the addressable video memory. The panning position is defined by means of the STAGE instruction SetPanPos $(x, y)$.

We can now integrate the overlay and panning operations, thus creating a window or mask moving across the scene in synchrony with the eyes. The scene that we want to present to a viewer is held in a constant, external image supplied to the Vista. The mask (window) that we want to move across this scene is stored as an opaque (transparent) region in the internal image, which is otherwise transparent (opaque). The addressable resolution for this internal image is set to be twice as wide and twice as high as the displayable resolution, and the center of the mask (window) is aligned with the center of the addressable video memory. As is shown in Figure 3, we can now pan in addressable video memory, which amounts to moving a large overlay over a smaller stable image. The visible effect of this operation consists of a series of shifts of the internally stored mask (window) across the entire height and width of the external image, which remains constant on the screen. Every time a new fixation position on the screen is registered, the panning

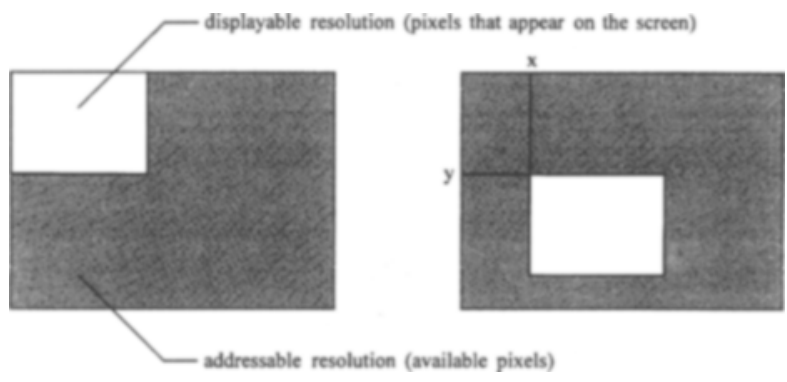

Figure 2. Panning the displayable resolution through the addressable resolution. The white rectangle represents the displayable resolution, which is output to the screen. The gray rectangle represents the addressable resolution. On the left, the upper left panning position ( 0 , $0)$; on the right, an arbitrary panning position $(x, y)$.

position is adjusted accordingly and the mask (window) is centered on the fixation position. Appearance of the mask (window) or scene can be terminated or initiated at any time simply by switching display modes. In the next section, a more detailed description is given of our implementation of the moving overlay method in a concrete experimental setup.

\section{A MULTIPLE VISTA EXPERIMENTAL SETUP}

In the overlay mode, the Vista composes an output image from the internal image stored in its own video memory and an externally generated image supplied to its video inputs. Any device capable of generating ana$\log$ RGB + sync PAL or NTSC interlaced video signals can provide that external image. We used a second Vista for this purpose (Figure 4), which, relative to a camera or VCR, offers greater flexibility in preparation, on-line selection, and timing of the sequence of images to be presented during an experiment. The two Vista boards were placed in two parallel-connected Intel 386 microcomputers. According to manufacturer specifications, however, up to eight boards can be placed in one computer (Truevision, 1989), which would further simplify the implementation of the moving overlay method. Although multiple Vista boards can function independently, they do need to employ the same video timing. Synchronization is achieved by designating one "master" Vista, which generates the synchronization signals for all other "slave" Vistas, as well as for the monitor. This is achieved by genlocking the slaves (STAGE instruction: EnableGenLock) to the master (STAGE instruction: DisableGenLock). In the moving overlay setup, the master then generates the stable scene that will be presented to the viewer while the slaves contain overlays. Transparency and movement of these overlays can be controlled independently.

In our two-Vista setup, the boards were genlocked by using interlaced, NTSC video timing $(60-\mathrm{Hz}$ field refresh rate) with a 16-bit pixel depth. Other video modes may be selected as well. However, use of the video in- 


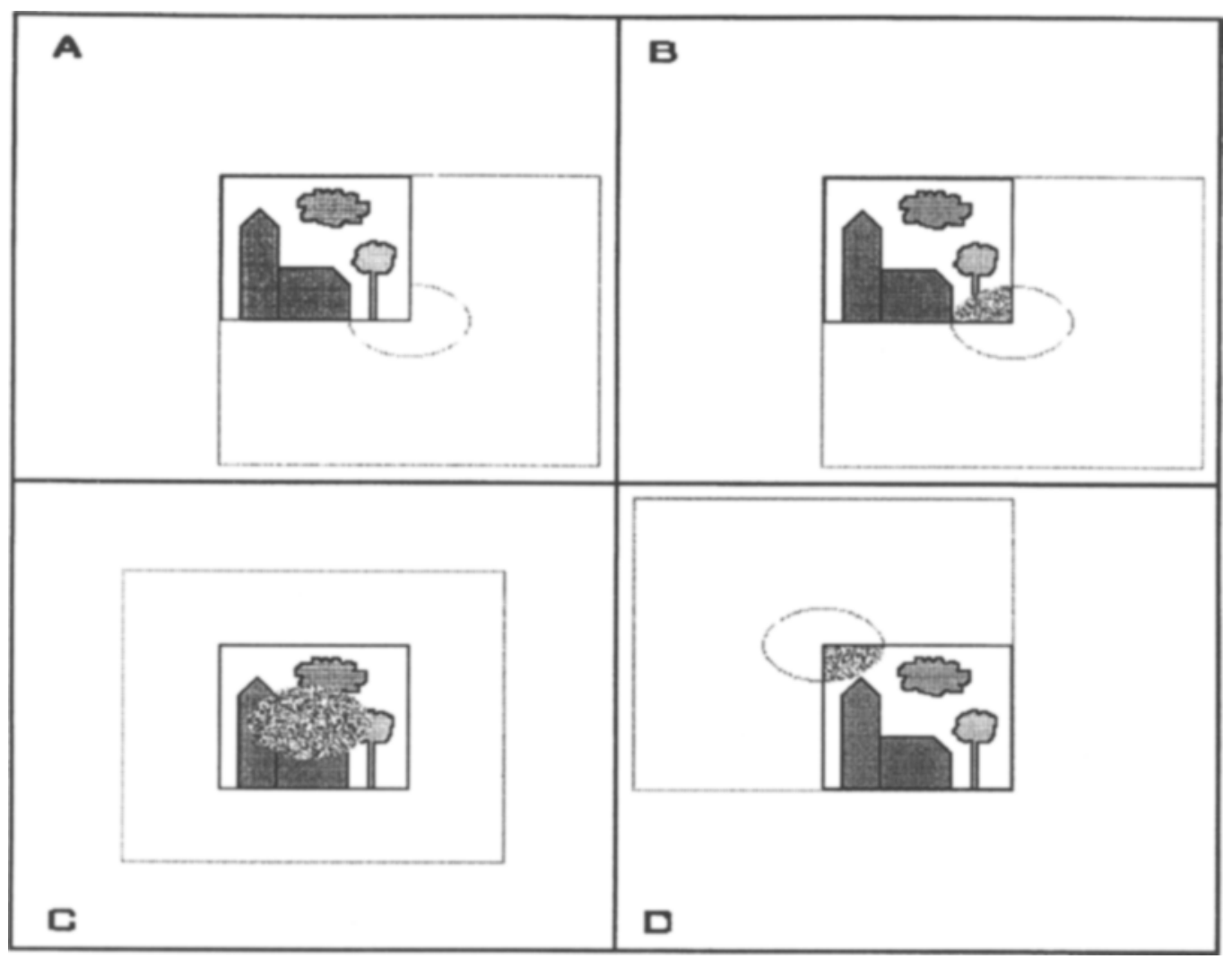

Figure 3. A moving mask based on the moving overlay technique. In the live mode (A), the stimulus generated by an external video source is output to the screen represented by the small dark-lined rectangle. Switching to the overlay mode (from panel A to panel B) enables the mask stored in the Vista addressable video memory, which is represented by the large, light-lined rectangle. Panels B, C, and D show the stimulus + mask in different panning positions.

puts on the Vista limits the pixel presentation rate, owing to a maximum analog-to-digital sample rate of 14.3 MHz. This forces us to use interlaced video, which implies that the time to change a whole display (both even and odd fields) is twice that for noninterlaced video.

The $4 \mathrm{Mb}$ of Vista video memory can only be organized in three configurations: 1,024 rows of $4 \mathrm{~K}, 2,048$ rows of $2 \mathrm{~K}$, or 4,096 rows of $1 \mathrm{~K}$. To create an addressable matrix that is twice as wide and high as the displayable matrix, a memory configuration is required which accommodates $y$ rows of $x$ bytes each, where

$$
y=2 \text { (displayable height), }
$$

and

$$
x=2(\text { displayable width })(\text { pixel depth/8). }
$$

This implies that in standard NTSC $(756 \times 486$ display resolution), one can maximally use 16 bits to define the appearance of each pixel.

When the master and slave Vistas have been synchronized, the full addressable resolution on the master Vista can be used to store a number of display pages.
The potential number of pages depends on the defined pixel depth and decreases as a greater palette of colors is desired. In NTSC mode this means that 10 pages are available in 8-bit resolution, 4 pages in 16-bit resolution, and 2 pages in 32-bit resolution. The different pages can each hold a background to be used in an overlay or can simply contain subject instructions or a pretrial fixation point. A fast switch between pages is realized by the SetPanPos instruction, thus enabling an effective integration of virtual screen and overlay techniques.

We are presently using the two-Vista setup in a series of experiments during which subjects freely explore complex line drawings of real-world scenes in order to count specific objects in these scenes. The scenes are presented in interlaced, standard NTSC mode on a Barco $6351 \mathrm{CRT}$ and subtend $16^{\circ} \times 12^{\circ}$ of visual angle at a distance of $125 \mathrm{~cm}$. Eye position is sampled every millisecond with a Generation 5.5 dual-Purkinje-image eyetracker (Crane \& Steele, 1985), and an on-line decision is made for every incoming measurement as to whether the eye is fixating or saccading. Eye position and state are continuously communicated to an Intel 386 personal computer that controls stimulus presentation by 


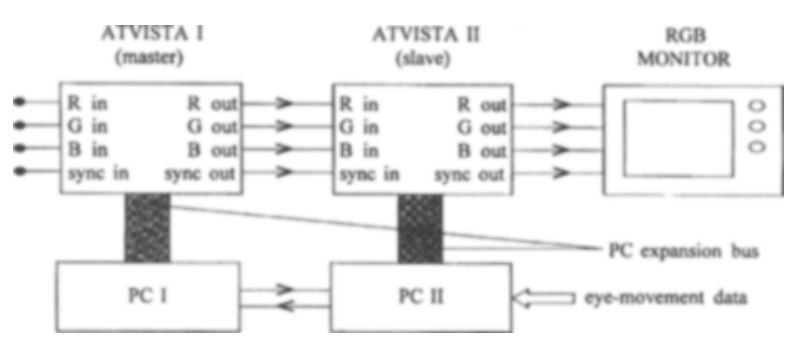

Figure 4. Experimental setup, using two Vistas. The video inputs of the master Vista are not used. The external input to the slave Vista is an internal image generated on the master Vista.

means of the Vista boards. A typical trial in these experiments consists of the following events: Subjects fixate a cross (Display Page 1 on the master Vista). When the tracker detects a steady gaze at the cross, the computer initiates presentation of the scene by immediately switching to Display Page 2 on the master Vista. Subjects finish scene exploration by pressing a response key, effecting an instantaneous switch to Display Page 3 on the master Vista, which contains response instructions. While the subject explores the scene, an elliptical noise mask or window (stored on the slave Vista) moves in synchrony with the eyes. Present manipulations include mask size $\left(1.5^{\circ} \times 1^{\circ}\right.$ vs. $2.5^{\circ} \times 1.7^{\circ}$ of visual angle $)$ and mask onset delay measured from fixation onset (15$120 \mathrm{msec}$ ). Future manipulations may include mask content (e.g., graded masks or parts of another scene instead of a noise mask), mask and window shape, and informational content outside the window. Importantly, mask content and informational content outside the window are fixed for the duration of a trial and can only be changed between trials.

\section{PERFORMANCE}

In principle, changing the contents of an entire screen requires one full refresh cycle. In interlaced video, this is accomplished in two vertical retraces: one to write the even lines on the display (the even field), and one to write the odd lines (the odd field). In our application, which uses NTSC interlaced, this whole process takes $2(1 / 60 \mathrm{sec})$, or $33.3 \mathrm{msec}$. However, it seems reasonable to assume that replacing every other line of a displayed stimulus by a pattern mask will already be sufficient to disrupt the perception of that stimulus. Conversely, replacing every other line of a blank screen by the image contained in a window is probably sufficient to support the percept of the stimulus in the window. Hence, our application involves a minimum 16.7-msec interval (i.e., one field refresh) to change the informational content of a complete screen. Unfortunately, the actual delay between the instruction to change and the completion of that change can be considerably longer if one uses the standard STAGE panning instruction, because SetPanPos will always wait for the electron beam to reach the top of the screen before it initiates a display change.
Using the SetPanPos instruction in NTSC interlaced video, we measured the delay between panning request and appearance of the first scan line with new panning coordinates and found intervals ranging from 2.0 to $25.0 \mathrm{msec}$ (van Diepen, 1993). ${ }^{2}$ To cut down on this delay, we developed a new reposition command that immediately starts to change panning position regardless of the scanning position of the electron beam (van Diepen, 1993). ${ }^{3}$ The execution time of this routine is $1.3 \mathrm{msec}$ and is followed immediately by the desired display change from the current scan line onward. Thus, in NTSC video, this new routine will never require more than $18.0 \mathrm{msec}$ for the display of every other scan line at the new panning coordinates. The exact repositioning lag-the time required to command a position change for a mask (window) and then write every other line of that mask (window) at the new position-will of course still be variable. This is because the position of the electron beam at the time of the reposition command and the intended position of the mask (window) are unrelated. For a sizable mask with a height of 100 lines presented in standard NTSC interlaced, this means that the lag will vary between 4.5 and $18.0 \mathrm{msec}$, with a mean of $11.2 \mathrm{msec}$. However, this random variability can be traced by directly reading scan line positions from the GSP registers (van Diepen, 1993). In this manner, the exact moment of appearance of a relocated mask (window) can be determined and stored along with the eyemovement data, thus allowing for precise estimates of the chronometry of masking (windowing) effects on perceptual performance.

\section{CONCLUSION}

We have proposed a method for realizing fast, carefully timed and positioned movement of a mask or window across a stable background. This moving overlay method is built on the ability of the Vista graphics board to display a full-color, high-resolution image that is composed of two distinct sources: a stable background and a movable, partly transparent foreground. Possibilities for image input to the application are diverse: Instead of the master Vista, any other image source capable of providing RGB + sync interlaced video signals can be used. In addition, shape, size, and content of the visible foreground and background portions of the displayed image can be varied at will. Hence, with the position of the foreground brought under eye-movement control, the basis is provided for a wide range of experiments aimed at unraveling the chronometry and spatial extent of pictorial information processing.

\section{REFERENCES}

Boyce, S. J., \& PollatseK, A. (1992). Identification of objects in scenes: The role of scene background in object naming. Journal of Experimental Psychology: Learning, Memory, \& Cognition, 18, 531-543.

Crane, H. D., \& Steele, C. M. (1985). Generation-V dual-Purkinjeimage eyetracker. Applied Optics, 24, 527-537. 
De Graef, P. (1992). Scene-context effects and models of real-world perception. In K. Rayner (Ed.), Eye movements and visual cognition: Scene perception and reading (pp. 243-259). New York: Springer-Verlag.

De Graef, P., De Troy, A., \& D'Ydewalle, G. (1992). Local and global contextual constraints on the identification of objects in scenes. Canadian Journal of Psychology, 46, 490-509.

Dixon, P. (1991). Tachistoscopic display using color-lookup-table display devices. Behavior Research Methods, Instruments, \& Computers, 23, 380-386.

FinLEY, G. P. (1991). A VGA tachistoscope. Behavior Research Methods, Instruments, \& Computers, 23, 546-547.

IsHIDA, T., \& IKEDA, M. (1989). Temporal properties of information extraction in reading studied by a text-mask replacement technique. Journal of the Optical Society of America, 6, 1624-1632.

LofTUS, G. R., \& GINN, M. (1984). Perceptual and conceptual masking of pictures. Journal of Experimental Psychology: Learning, Memory, \& Cognition, 10, 435-441.

Matin, E. (1974). Saccadic suppression: A review and analysis. Psychological Bulletin, 81, 899-917.

MCCONKIE, G. W., \& RAYNER, K. (1975). The span of the effective stimulus during a fixation in reading. Perception \& Psychophysics, 17, 578-586.

PoultoN, E. C. (1962). Peripheral vision, refractoriness, and eye movements in fast oral reading. British Journal of Psychology, 53, 409-419.

Rayner, K., Inhoff, A. W., Morrison, R. E., Slowiaczek, M. L., \& BERTERA, J. H. (1981). Masking of foveal and parafoveal vision during eye fixations in reading. Journal of Experimental Psychology: Human Perception \& Performance, 7, 167-179.

RAYNer, K., \& Pollatsek, A. (1989). The psychology of reading. Englewood Cliffs, NJ: Prentice-Hall.

SAIDA, S., \& IKEDA, M. (1979). Useful visual field size for pattern perception. Perception \& Psychophysics, 25, 119-125.

TEXAS INSTRUMENTS, INC. (1986). TMS34010 user's guide. Houston, TX: Author.
TRUEVISION, INC. (1988). ATVista videographics adapter technical reference manual. Indianapolis, IN: Author.

TRUEVISION, INC. (1989). STAGE Coach, reference manual for STAGE. Indianapolis, IN: Author.

VAN DIEPEN, P. M. J. (1993). Use of the ATVista videographics adapter in visual perception research (Psychological Report No. 154). University of Leuven, Belgium, Laboratory of Experimental Psychology.

VAn Rensbergen, J., \& De Troy, A. (1993). A reference guide for the Leuven dual-PC controlled Purkinje eyetracking system (Psychological Report No. 145). University of Leuven, Belgium, Laboratory of Experimental Psychology.

WitTebrood, J. E., WANSINK, M. G., \& DE Weert, C. M. (1981). A versatile colour stimulus generator. Perception, 10, 63-69.

\section{NOTES}

1. The execution time of the command to switch between display modes is $0.8 \mathrm{msec}$ for NTSC interlaced video, following which the actual change on the screen starts at the current scan line. This may result in a brief impression of a split screen, with different images above and below the scan line at which the switch started having an effect. The split-screen effect will disappear during the next screen refresh.

2. With the SetPanPos instruction, the first scan line with new panning coordinates is always the top line of either the even or the odd field. The variance in the delay depends on the electron beam position and the old and new panning coordinates.

3. The timing and panning routines developed for the moving overlay method can also benefit applications that use a single Vista board as a general-purpose, multichannel tachistoscope.

(Manuscript received May 5, 1993; revision accepted for publication January 21, 1994.) 